\title{
Dynamic Simulation of Splashing Fluids
}

\author{
James F. O'Brien and Jessica K. Hodgins \\ College of Computing \\ Georgia Institute of Technology \\ Atlanta, GA 30332-0280 \\ [obrienj | jkh]@cc.gatech.edu
}

\begin{abstract}
In this paper we describe a method for modeling the dynamic behavior of splashing fluids. The model simulates the behavior of a fluid when objects impact or float on its surface. The forces generated by the objects create waves and splashes on the surface of the fluid. To demonstrate the realism and limitations of the model, images from a computergenerated animation are presented and compared with video frames of actual splashes occuring under similar initial conditions.
\end{abstract}

\section{Introduction}

The world is filled with natural phenomena that are remarkable in their form and movements: a tree blowing in the wind, lightning arcing across the sky and the simple elegance of ripples in a pool of water. We, along with others in the computer graphics community, are attempting to emulate nature's splendor in our computer animations. Our approach to this problem is to add realism to computer-generated images and animations through the use of models and simulations that approximate the physical laws of nature.

While many natural phenomena appear simple, the true complexity of these behaviors is considerable. For example, a rock falling into a pool of water involves complex interactions between the rock and the water. The rock strikes the pool's surface, displacing water and creating a disturbance that travels outward from the impact as it forms waves on the surface. Fluid that is displaced with sufficient force breaks free of the pool and flies through the air as spray. Cohesion between water molecules causes the formation of sheets of water, and air forced beneath the surface generates foam and bubbles.

Computer animations have been produced by skilled animators manipulating models of a computergenerated world using their intuition about the real or

From the proceedings of Computer Animation '95, pages 198205. Held April 19-21, 1995, in Geneva, Switzerland.

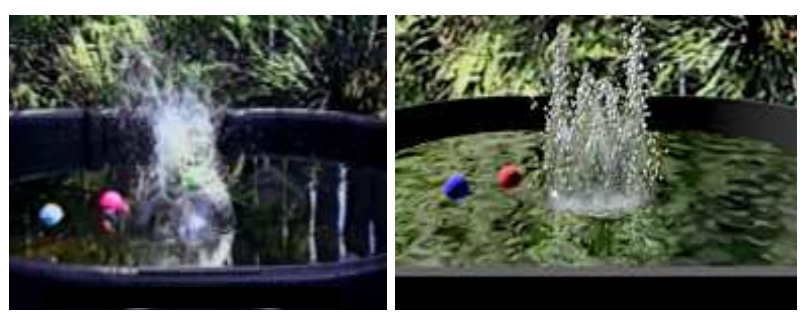

Figure 1: Comparison between a video image of a physical splash and a synthetic image generated using dynamic models of the fluid and the impacting object.

imaginary physical laws of the animated universe. To provide the animator with higher level tools for producing realistic computer animations of natural phenomena, researchers have investigated the use of dynamically based simulations. By modeling the physical effects of natural forces, the modeled systems can display realistic behavior without explicit specification of the movement for each object. Unfortunately, physically correct simulations of complex natural phenomena are often computationally expensive, requiring many hours of computation time to generate only a few seconds of motion.

In the following sections, we describe a method for modeling the dynamic behavior of a fluid. The simulation method allows animation of impacts to the surface of the fluid, splashes, and the waves that arise as a result of an impact. The simulation also models the behavior and effects of objects floating on the surface of a fluid. Because our goal is to provide a method suitable for computer animation, we have compromised accuracy for reduced computation time. Nonetheless, these methods model many of the visual effects seen in the real world, as demonstrated by the images in Figures 1 and 7. 


\section{Background}

Fluids have been simulated using a number of different techniques. One of the most accurate is to solve the 3D Navier-Stokes equations describing the fluid system [4]. This approach requires dividing the space that the fluid occupies into a lattice of cells and computing the behavior of the fluid as it moves through the cells. Although this approach is well suited for scientific simulations requiring a high level of detail, the lower bound of the computational cost increases with the cube of the model's resolution, making it impractical to use this method for interactive computer animation.

Particle systems have been used to model waterfalls and other forms of falling water [9]. The dynamics equations used in these simulations model particles falling under the influence of gravity without modeling interactions among the particles. Phenomena such as spray or loosely packed water droplets are easily modeled by this technique. Systems that allow particle interactions have been used to model fountains of fluid and amorphous solids [5]. While a large volume could potentially be modeled with interacting particles, the number of particles needed to fill a volume will grow with the cube of the resolution of the model, and despite the simplified equations for the dynamics this method can be computationally expensive for a large volume of particles.

Fournier and Reeves [2] and Peachey [7] implemented techniques that model the movement of explicit waves over the surface of a fluid. They used functions that describe the shape of a wave to displace a model representing the free surface of the fluid. Spray from the top of the wave crests is modeled using a particle system. Although these methods work well for modeling the propagation of wave-trains, it is more difficult to apply them to simulations where no explicit set of wave-fronts exist, and this approach does not specify how wave-trains should be created as a result of an impact to the surface.

An efficient approach to the problem of simulating solid volumes of water is to use a system that models a simplified subset of the fluid dynamics. Kass and Miller [3] used this approach and represented the surface with a height field, modeling the flow between adjacent columns of fluid. With this method, surface artifacts, such as waves, do not need to be explicitly specified because they arise naturally from the physical conditions occurring within the system. The model can be used for large volumes of fluid without incurring huge computational costs as the size of the height field is proportional to the square, rather than the

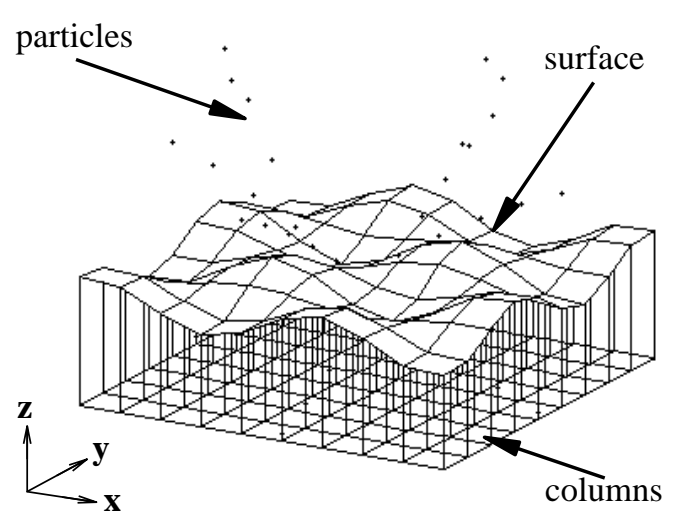

Figure 2: The fluid model is a three part system containing subsystems for volume, surface and spray. These subsystems interact to model the dynamics of the fluid body.

cube, of the resolution. Chen and Lobo implemented a similar system that uses a simplification of the 3D Navier-Stokes Equations to model fluid behavior in the presence of moving obstacles [1].

Although these systems provide a good set of tools for modeling the behavior of waves and falling water, we would like to model the interaction of water with other objects. Our method extends the approach described by Kass and Miller to allow a wider range of behaviors such as the splashes caused by impacts and the behavior of floating objects.

\section{Simulation Model}

To simulate the surface behavior of a body of fluid, we use a three-part system where each subsystem corresponds to a physical area of the fluid body: the main volume, the free surface of the fluid, and disconnected components of the fluid (spray). Each subsystem models the effects of one aspect of the overall behavior of the fluid. Taken together, the subsystems, along with the interfaces between them, form the basis for the model (Figure 2).

\subsection{Volume Model}

To model the volume that makes up the main body of the fluid, we use a formulation that divides the body into a rectilinear grid of connected columns. The model assumes vertical isotropy within the system and all fluid properties within a column are assumed to be constant. Flow between these columns occurs through a set of virtual pipes that connect adjacent columns. Pipes connect columns along the diagonals as well as along the axis-aligned directions (Figure 3).

The equations to determine flow in the pipes are 


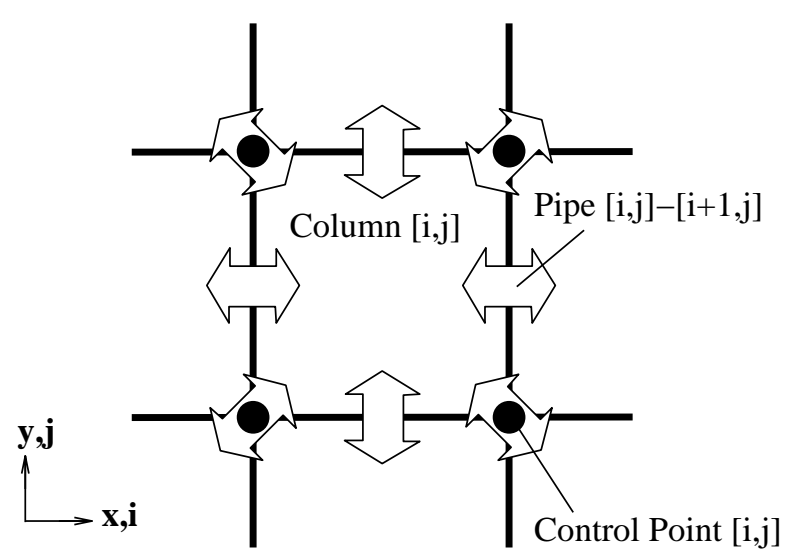

Figure 3: Each vertical column is connected to its eight neighbors through a set of directional horizontal pipes. The control points for the surface are sampled between the columns so that the lines of the surface grid separate adjacent columns.

derived from the physical laws for hydrostatic pressure. The static pressure of a column in the grid at position $[i, j], H_{i j}$, is

$$
H_{i j}=h_{i j} \rho g+p_{0}
$$

where $\rho$ is the density of the fluid, $g$ is the acceleration due to gravity, $p_{0}$ is the atmospheric pressure in the system, and $h_{i j}$ is the height of the column at $[i, j]$. The height of a column is related to the volume of that column, $V_{i j}$, by

$$
h_{i j}=\frac{V_{i j}}{d_{x} d_{y}}
$$

where we approximate the cross-sectional column area with the product of the nominal distance between mesh grid points in the $x$ and $y$ directions, $d_{[x \mid y]}$. When used to determine the flow, equation (1) is an approximation because it relies on the assumption that the fluid is not moving rapidly [6].

In addition to the pressure due to gravity, we model external forces to simulate the impact of objects on the surface of the fluid. These forces are described in Section 4 , but for now we include an additional term, the external pressure $E_{i j}$, and rewrite equation (1) for total pressure $P_{i j}$ :

$$
P_{i j}=h_{i j} \rho g+p_{0}+E_{i j} .
$$

As previously mentioned, each column is connected to its eight neighbors by a set of virtual pipes. A flow velocity is maintained for each pipe in the system and at each time step a force accelerates the flow of fluid in the pipe. The force is determined by measuring the pressure differential, $\Delta P_{i j \rightarrow k l}$, across the pipe between column $[i, j]$ and one of its eight neighbors, $[k, l] \in \eta_{i j}$. Substituting into the elementary equation $F=m a$, we can derive the acceleration of the fluid in a pipe, $a_{i j \rightarrow k l}$ :

$$
a_{i j \rightarrow k l}=\frac{c\left(\Delta P_{i j \rightarrow k l}\right)}{m}
$$

where $c$ is the cross-sectional area of the pipe and $m$ is the mass of the fluid in the pipe. Expressing the mass of the fluid in a pipe of length $l$, as $m=\rho c l$, and assuming atmospheric pressure to be constant, equation (4) can be rewritten:

$$
a_{i j \rightarrow k l}=\frac{\rho g\left(h_{i j}-h_{k l}\right)+E_{i j}-E_{k l}}{\rho l} .
$$

Assuming that the acceleration is constant over some time period, $\Delta t$, the flow in the pipe, $Q_{i j \rightarrow k l}$, is

$$
Q_{i j \rightarrow k l}^{t+\Delta t}=Q_{i j \rightarrow k l}^{t}+\Delta t\left(c a_{i j \rightarrow k l}\right)
$$

and the net volume change of a column during a time interval is

$$
\Delta V_{i j}=\Delta t \sum_{k l \in \eta_{i j}}\left[\frac{Q_{i j \rightarrow k l}^{t+\Delta t}+Q_{i j \rightarrow k l}^{t}}{2}\right] .
$$

In order to conserve volume within the system, some constraints must be added to the system. Because the fluid is incompressible, the flow at one end of a pipe must be equal in magnitude with opposite sign to the flow at the other end:

$$
\forall i j: \forall k l \in \eta_{i j}: Q_{i j \rightarrow k l}=-Q_{k l \rightarrow i j} .
$$

A negative volume does not make physical sense, and the following inequality ensures that the volume in all columns remain positive:

$$
V_{i j}^{t+\Delta t} \geq 0 \Longleftrightarrow V_{i j}^{t} \geq-\Delta V_{i j}^{t} .
$$

To keep equation (9) true at the end of each integration step, the volume of each column is tested. If a column has a negative volume, then all pipes that are removing fluid from that column are scaled back. This procedure is repeated until all columns in the grid have a positive volume.

The columns on the outer edges of the grid have pipes that lead out of the area where columns are defined. Because no columns exist at the other end of the virtual pipe, flow conditions must be specified to model boundary conditions. To model a barrier, such as a wall, the flow is set to zero. Phenomena such as fluid sources or sinks can be created by setting the flow to a positive or negative constant. 


\subsection{Surface Model}

The surface subsystem allows external objects to interact with the fluid system. Objects that collide with or float on the surface exert forces on the surface model. These forces are propagated as external pressure to the volume subsystem. The vertical positioning of the surface elements is determined by the volume of the columns.

The model for the surface subsystem is a rectilinear grid of control points that define a mesh. The control points of the mesh map onto the column grid of the volume model so that the control points are sampled between adjacent columns (Figure 3). The vertical position of a grid point, $z_{i j}$, is determined by averaging the height of the four columns surrounding the grid point:

$$
z_{i j}=\frac{h_{i, j}+h_{i, j+1}+h_{i+1, j}+h_{i+1, j+1}}{4} .
$$

Forces applied to each control point are reformulated as an external pressure, $E_{i j}$, applied to the columns contacted by that control point:

$$
E_{i j}=-\frac{f_{e}}{4 d_{x} d_{y}}
$$

where $f_{e}$ is the force applied to the control point. A downward force (negative $z$ direction) will result in an increase in external pressure, hence the negative sign. Grid points contact four columns and the external pressure is evenly distributed across these columns.

\subsection{Spray Model}

To model droplets that are disconnected from the main body of the fluid, we implemented a particle system using techniques similar to those described by Reeves [8] and Sims [9]. Particles are created when the upward velocity of a portion of the surface exceeds a threshold ${ }^{1}$. Because the vertical position of a column is determined by the volume of fluid in the column, the column's vertical velocity can be determined by the rate of change in volume (the net flow into the column from its neighbors):

$$
\dot{h}_{i j}=\sum_{k l \in \eta_{i j}} \frac{Q_{k l \rightarrow i j}}{d_{x} d_{y}} .
$$

The vertical velocity at the surface is

$$
\dot{z}_{i j}=\frac{\dot{h}_{i, j}+\dot{h}_{i, j+1}+\dot{h}_{i+1, j}+\dot{h}_{i+1, j+1}}{4}
$$

\footnotetext{
${ }^{1}$ The value for the threshold can be adjusted to modify the appearance of the splash. Lower threshold values will cause more particles to be generated over a larger area. The values used to generate the images in this paper were between 2.0 and $2.5 \mathrm{~m} / \mathrm{s}$.
}

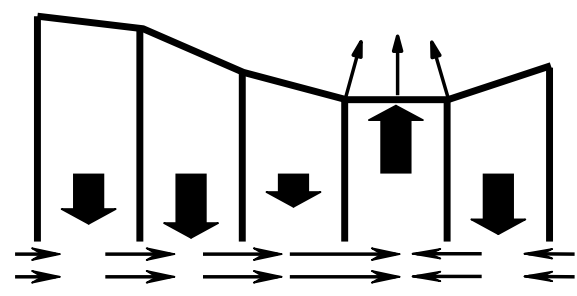

Figure 4: Initial velocity of ejected particles. The horizontal arrows at bottom indicate direction of flow between columns, the thick vertical arrows indicate upward velocity in columns and the thin arrows at top indicate the velocity vectors for ejected particles.

and the horizontal velocity of the fluid at the surface is

$$
\begin{gathered}
\dot{x}_{i j}=\frac{Q_{i, j \rightarrow i+1, j}+Q_{i, j+1 \rightarrow i+1, j+1}}{2} \\
\dot{y}_{i j}=\frac{Q_{i, j \rightarrow i, j+1}+Q_{i+1, j \rightarrow i+1, j+1}}{2} .
\end{gathered}
$$

When an area of the surface has an upward velocity greater than the threshold, particles are distributed uniformly over that area and the initial velocities for the particles are interpolated from the surface velocities (Figure 4).

To conserve the total volume in the system, the volume of each particle is subtracted from the column from which it was created. Once created, the particles fall under the influence of gravity and do not interact with each other.

Particles are removed from the system when they fall back onto the surface, and the volume of each particle is added to the column that absorbed it. Particles that fly away from the fluid body are destroyed when they encounter a ground plane or strike some other object.

\section{External Objects}

When an object collides with the fluid surface, we model the motion of the fluid and of the object by computing the forces resulting from the collision and applying them to the fluid surface and the object. The behavior of an object with mass $m$, at a location $s_{0}$, moving at a velocity $\dot{s}$, is described by:

$$
s=s_{0}+\dot{s} t+\frac{1}{2}\left(g+\frac{f_{o}}{m} \Delta t^{2}\right)
$$

provided that the force, $f_{o}$, and the acceleration, $g$, remain constant over the time interval.

The force acting on the fluid is equal in magnitude with opposite sign to the force acting on the object 
$\left(f_{o}=-f_{e}\right)$. If we assume that the object remains in contact with the surface and does not bounce off, then the location of the mass is determined by the location of the surface. The force acting at the impact could be computed by solving the nonlinear system derived from equation (16) and the fluid model system.

The force, $f_{o}$, is bound in the range $\left[0, f_{\max }\right]$, where $f_{\max }$ is the force that would cause the mass to have a final position equal to the initial position of the surface:

$$
f_{\max }=m\left(\frac{2\left(z-s_{0}-\dot{s} t\right)}{t^{2}}-g\right) .
$$

A search could be done to find the force that would minimize the difference between the final position of the mass and the surface.

An alternative solution that exchanges accuracy for speed is to select $f_{o}$ in the range $\left[0, f_{\max }\right]$ based on a heuristic measure of the expected deceleration of the mass as it strikes the surface, and perform the integration on the fluid system using the approximated value. Thus objects with a small impact face would have lower values, and objects with a large impact face would have larger values. We used this method to produce the images for this paper.

\section{Results and Conclusions}

In developing our simulation model, we made several approximations to simplify the calculations and to decrease the required computation time. These simplifications introduce errors into the final solution. We justify this approach by observing that computer animations do not generally require the same degree of accuracy as a scientific experiment. Furthermore, animation is often used to duplicate a known phenomenon, such as a ball falling into water, rather than an unknown phenomena, such as the air flow within an experimental jet engine. Because we are duplicating known phenomena, we can use recorded footage or physical intuition to "tune" the simulation, thus allowing the animator to create the desired effect while retaining many of the motion characteristics exhibited by water in the real world. For example to improve the correspondence shown in Figure 7 we adjusted the time during which the falling object was in contact with the water's surface. Other adjustable parameters include the number of particles to be spawned by portions of the surface that have exceeded the threshold, the volume of the ejected particles and the amount of damping used during flow integration.

The images in Figure 5 were rendered from data generated using the methods described above. These images show the splash that results when a physically based simulation of a rigid body model of a human diver enters the water after a 10 meter platform dive [10]. For the purposes of computing the intersection of the diver with the pool's surface, the human's body was approximated by a set of ellipsoids, each representing one of the diver's body parts. The pool covers an area of $16 \times 16$ meters represented with a $241 \times 241$ grid.

The system we have implemented runs at interactive speeds for simple problems (about one-tenth realtime for a $11 \times 11$ mesh), but the response time degrades as the resolution of the simulation mesh becomes finer and as multiple objects interact with the fluid surface. The images in Figure 6 show three balls floating on a 2 meter-square body of water. They were generated using data from a simulation on a $61 \times 61$ grid which took about 3 minutes actual time to complete 2 seconds of simulation time. The simulation for the images in Figure 5 took longer, a little over an hour for 20 seconds of motion, due to the higher resolution of the grid and the larger number of objects. These timings were performed on an SGI Indigo ${ }^{2}$ with an R4000 processor running at $100 \mathrm{mHz}$. Simulation time might be improved by using a non-uniform grid that would have a higher resolution in areas where impacts would occur and lower resolution elsewhere.

The full spectrum of behaviors exhibited by fluids is very complex, and the model we have described allows the relatively fast simulation of a subset of these behaviors including waves, impacts, splashes, floating objects and other behaviors that fit within the assumption of vertical isotropy. Phenomena that occur primarily due to vertical effects within the fluid, such as turbulence out of the horizontal plane, are beyond the scope of this model. Extending the model to include additional horizontal effects or phenomena such as flowing streams should be possible as would extensions to add foam or bubbles.

The visual appearance of the model could be improved by using a more sophisticated spray model that took into account the cohesion between drops of water such as the one described in [5]. Figure 7 shows a comparison between an actual splash and a simulation where we attempted to match the initial conditions of the actual splash. Although the simulated motion is similar to that appearing in the captured video images, a clear difference can be seen in the patterns formed by the ejected water droplets. The addition of a more sophisticated spray model would allow the formation of sheets in the water, improving the perceived realism of the splashes.

It is interesting to note that despite the obvious differences between the images of real and simulated 
splashes, the resulting computer animation appears realistic when viewed alone. An investigation as to what minimal set of motion qualities are essential for believability might enable further simplification and simulation at real-time speeds. Real-time execution would allow the inclusion of a simulation such as this into an interactive virtual reality system.

High resolution copies of the images in this paper and animated image sequences are available through the World Wide Web at the URL

$$
\text { "http://www.cc.gatech.edu/gvu/animation/ }
$$$$
\text { Animation.html". }
$$

\section{Acknowledgments}

The authors would like to thank Wayne Wooten and Debbie Carlson for their help in rendering images and Walter Patterson and Adrian Ferrier for their suggestions on simulation methods.

This project was supported in part by NSF Grant No. IRI-9309189 and funding from the Advanced Research Projects Agency.

\section{References}

[1] Chen, J. X. and Lobo, N. V., "Toward Interactiverate Simulation of Fluids with Moving Obstacles by Navier-Stokes Equations," CVGIP: Graphical Models and Image Processing, Vol. 54, No. 3, in press, 1995.

[2] Fournier, A. and Reeves, W., "A Simple Model of Ocean Waves," Proceedings of SIGGRAPH '86, in Computer Graphics, Vol. 20, No. 4, pp. 75-84, 1986.

[3] Kass, M., and Miller, G., "Rapid, Stable Fluid Dynamics for Computer Graphics," Proceedings of SIGGRAPH '90, in Computer Graphics, Vol. 24, No. 4, pp. 49-57, 1990.

[4] Knight, B., "Computer Modeling of a Thermal Inkjet Device," Seventh International Congress on Advances in Non-Impact Printing Technologies, Vol. 1, 1991.

[5] Miller, G., Pearce, A., "Globular Dynamics: A connected Particle System for Animating Viscous Fluids," Computers and Graphics, Vol 13, No. 3, pp. 305-309, 1989.

[6] Munson, B., Young, D., and Okiishi, T., Fundamentals of Fluid Mechanics, John Wiley and Sons: Canada, 1990.
[7] Peachey, D., "Modeling of Waves and Surf," Proceedings of SIGGRAPH '86, in Computer Graphics, Vol. 20, No. 4, pp. 65-74, 1986.

[8] Reeves, W., "Particle Systems-A Technique for Modeling a Class of Fuzzy Objects," Computer Graphics, Vol. 17, No. 3, pp. 359-376, 1983.

[9] Sims, K., "Particle Animation and Rendering Using Data Parallel Computation," Proceedings of SIGGRAPH '90, in Computer Graphics, Vol 24, No. 4, pp. 405-413, 1990.

[10] Wooten, W., and Hodgins, J., 1995. "Simulation of Human Diving," Proceedings of Graphics Interface '95, in press 1995. 

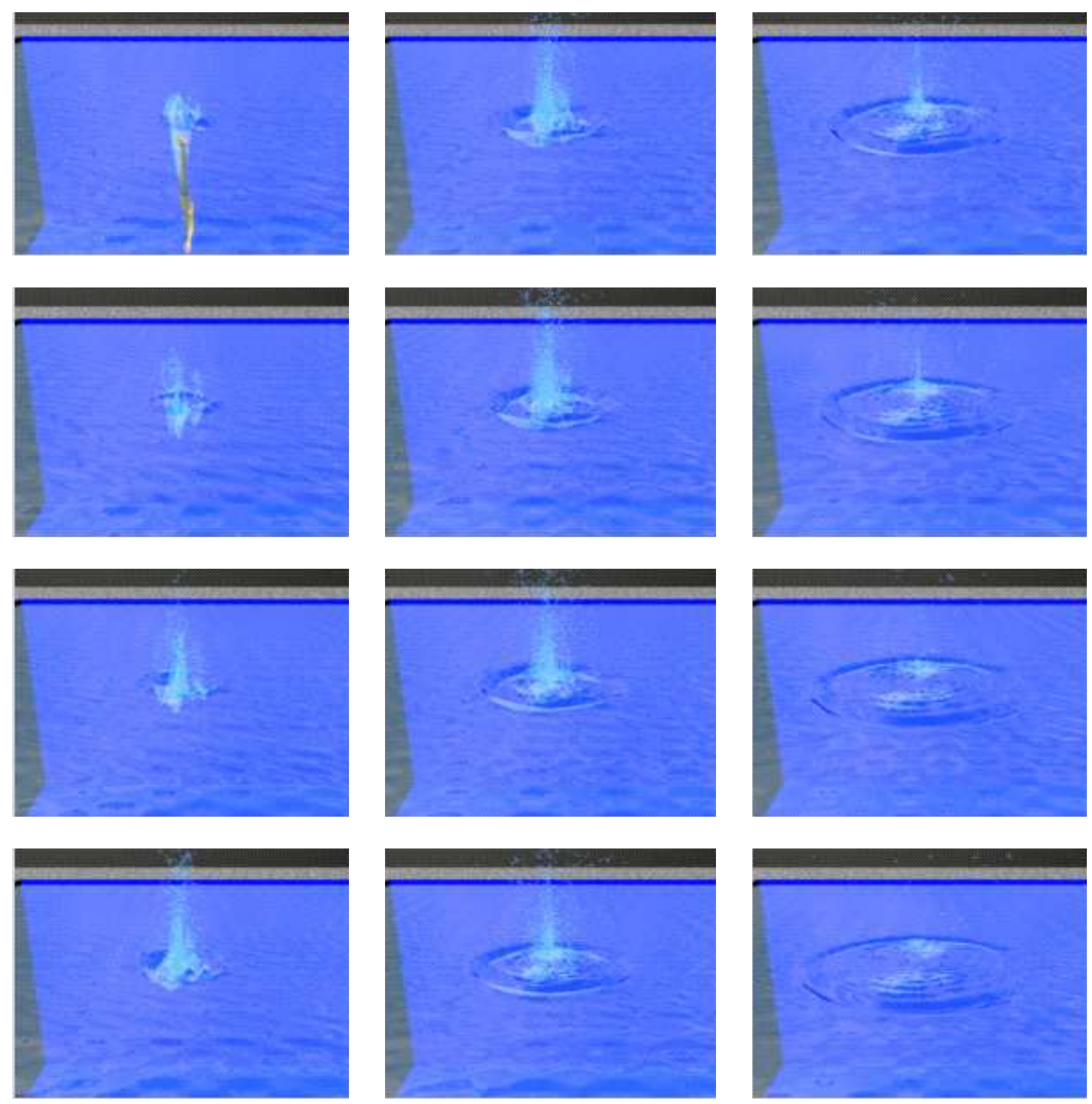

Figure 5: Image sequence from a computer-generated animation of a human diver entering the water. Frames progress from top to bottom and from left to right at intervals of 0.167 seconds. The motion of the diver was simulated using rigid body dynamics and control laws for a variety of platform dives[10]. The images were rendered with Photo-Realistic RenderMan on Silicon Graphics workstations. 

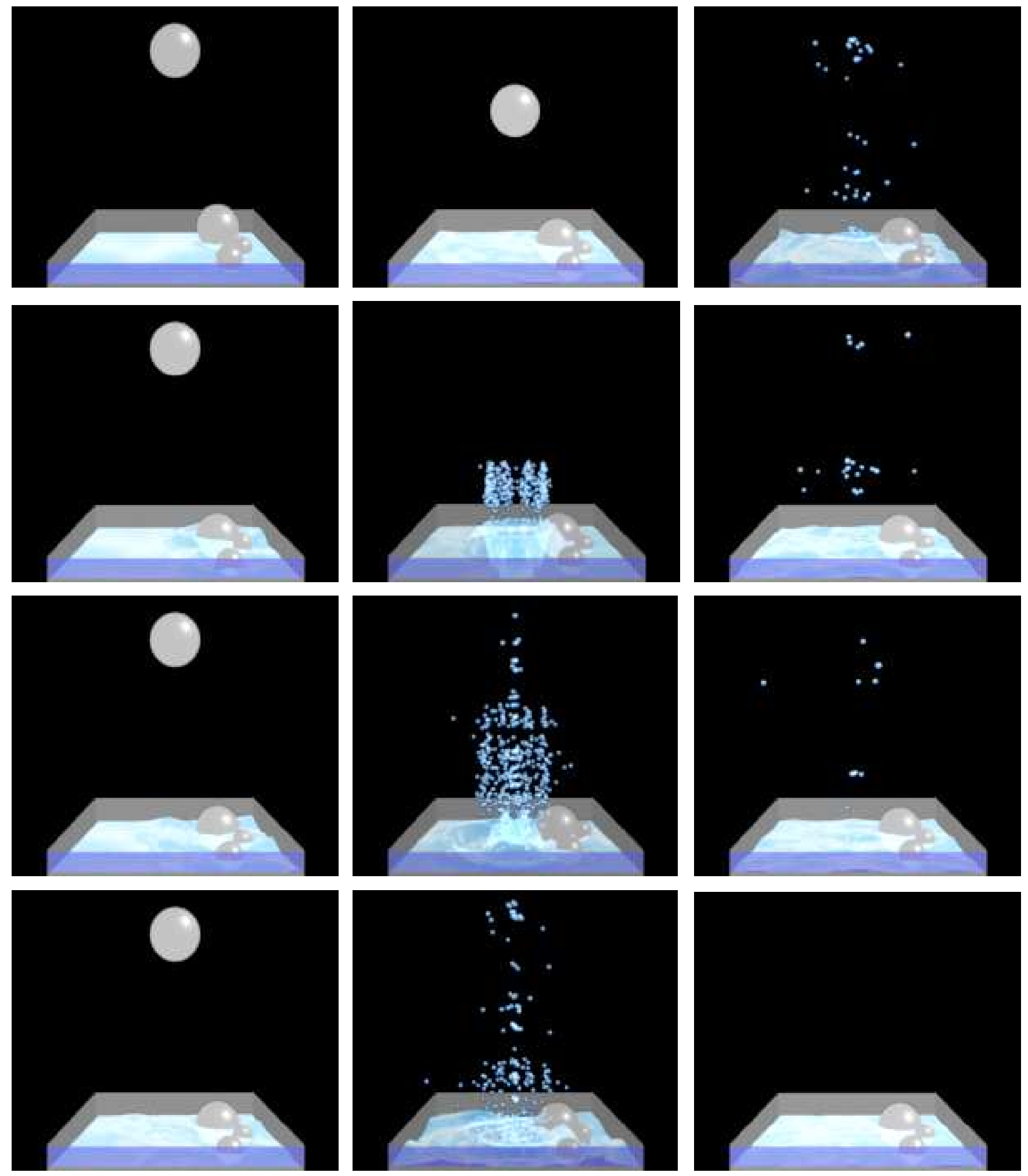

Figure 6: Objects floating on a fluid surface. Three balls of equal density but different sizes were dropped onto the fluid. After the balls settled onto the surface, a fourth ball of higher density was dropped into the fluid. The floating balls bob on the surface as the disturbance caused by the heavier ball moves past them. 

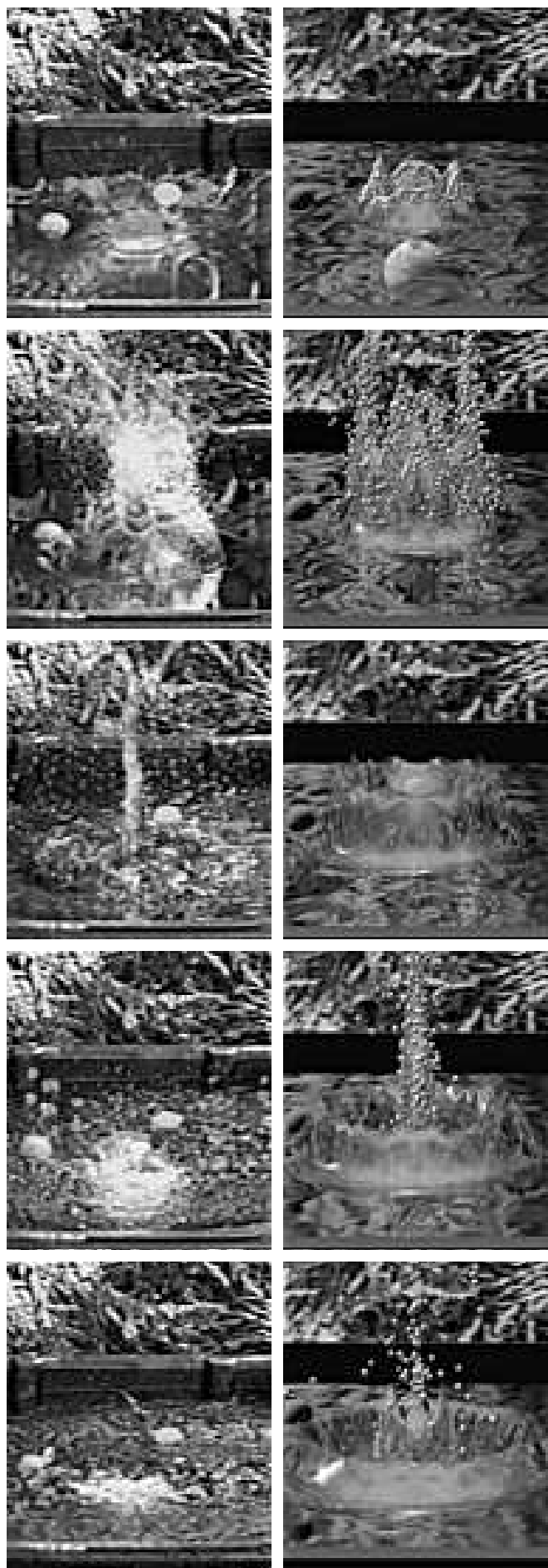
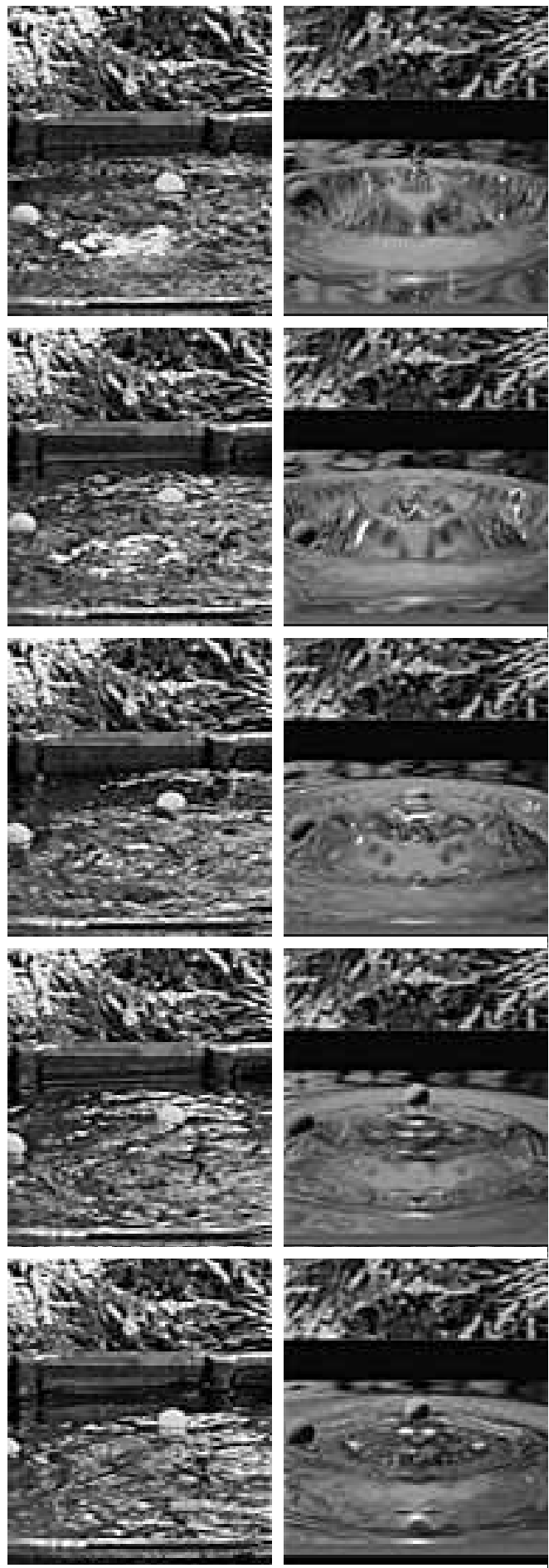

Figure 7: [See color plate.] A side-by-side comparison of video of an actual splash and images from a computer simulation in which we have attempted to match the initial conditions to those of the actual splash. The images proceed top to bottom and left to right and are spaced at intervals of 0.233 seconds. While the patterns formed in both image sequences are qualitatively similar, substantial differences exist between the two. For example, the video footage shows sheeting effects that are not present in the synthetic images. The vertical column that appears in the center of the actual splash in frame 3 does not appear in the simulated splash until frame 4 . Finally, the motion of the water in the video footage is more irregular. 

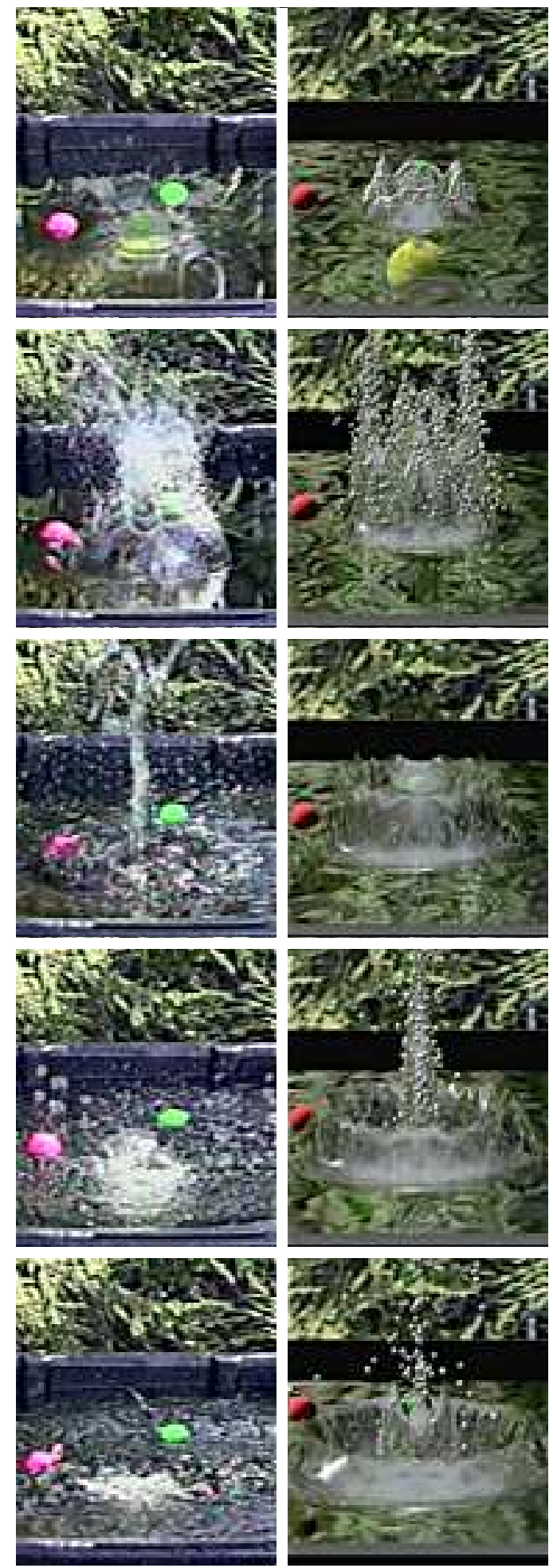
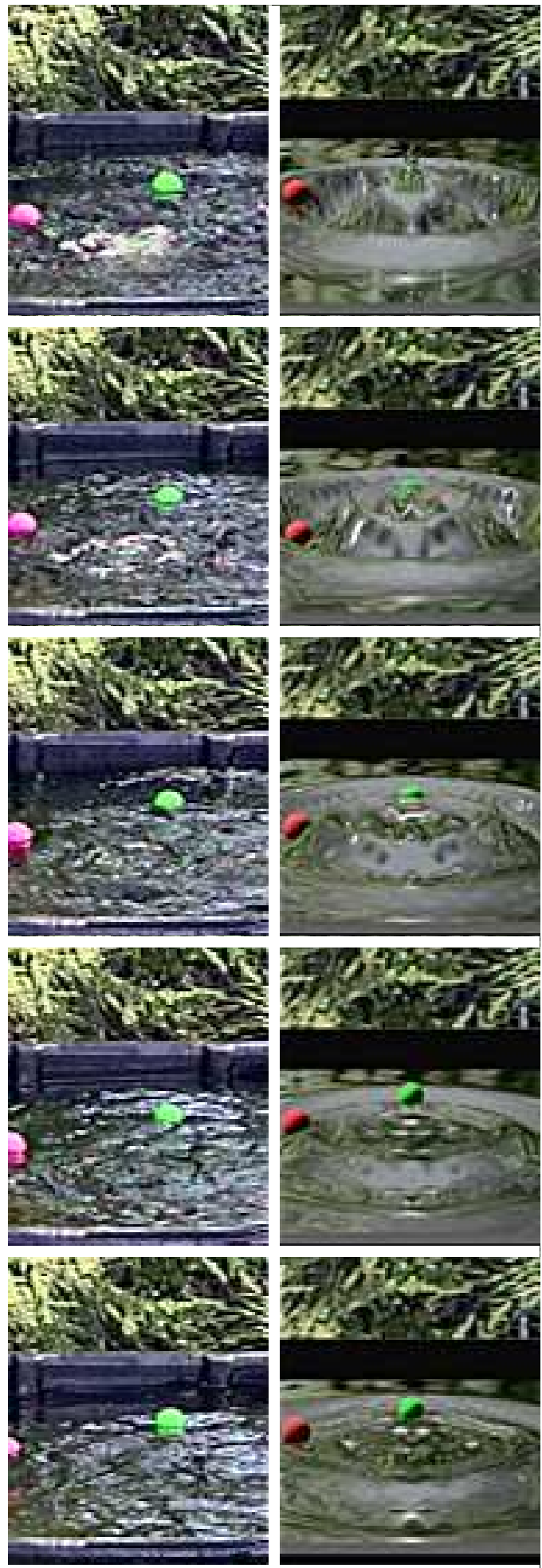

A side-by-side comparison of video of an actual splash and images from a computer simulation. 